\title{
An energy relaxation tolerant approach to quantum entanglement, information transfer, and gates with superconducting-quantum-interference-device qubits in cavity QED
}

\author{
Chui-Ping Yang ${ }^{1,2}$, Shih-I Chu ${ }^{2}$, and Siyuan Han ${ }^{1}$ \\ ${ }^{1}$ Department of Physics and Astronomy, University of Kansas, \\ Lawrence, Kansas 66045 \\ ${ }^{2}$ Department of Chemistry, University of Kansas, and Kansas Center \\ for Advanced Scientific Computing, Lawrence, Kansas 66045
}

\begin{abstract}
A scheme is proposed for realizing quantum entanglement, information transfer, CNOT gate, and SWAP gate with superconducting-quantum-interferencedevice (SQUID) qubits in cavity QED. In the scheme, the two logical states of a qubit are the two lowest levels of the SQUID. An intermediate level of the SQUID is utilized to facilitate coherent control and manipulation of quantum states of the qubits. The method presented here does not involve a real excitation of the intermediate levels during the operations. Thus, decoherence due to energy relaxation of intermediate levels is minimized. In addition, the present method does not require the adjustment of the SQUID level spacings, which simplifies the operation significantly.
\end{abstract}

PACS numbers: 03.67.Lx, 03.65.-w, 74.50.+r, 85.25.Dq, 42.50.Dv 
Cavity QED has been extensively studied to implement quantum information processing (QIP) with a variety of physical systems such as atoms, ions, quantum dots and Josephson junctions [1-5]. A well-known reason for this is that compared with those non-cavity proposals where significant overhead is needed for coupling distant qubits, the cavity-based schemes is preferable since the cavity mode acts as a "bus" that can mediate long-range fast interaction between any qubits, which enables one to perform two-qubit operations involving any desired pair of qubits.

Recently, a scheme has been proposed for obtaining quantum gates, information transfer, and entanglement with superconductor quantum interference devices (SQUIDs) in cavity QED [6]. The SQUID-cavity QED scheme may be among the most promising candidates for demonstrating QIP because (i) the cavity mode can mediate long-range and fast interaction between distant SQUID qubits, (ii) decoherence induced due to the external environment can be greatly suppressed since the cavity can be doubled as the magnetic shield for SQUIDs, (iii) and superconducting qubits have been demonstrated to have relatively long decoherence time [7-9]. In Ref. [6], the operations were performed by inducing transitions to the intermediate level $|a\rangle$ [see Fig. 1(a)] via microwave pulse and cavity field. However, though the cavity mode is not populated during the operation, the population of the SQUIDs in the intermediate levels is non-zero. Thus, energy relaxation of the intermediate level can cause decoherence during the operation. Another key point is that the operation in [6] requires rapid adjustments of level spacings of SQUIDs, which is undesirable in experiment, since tuning the SQUID level spacings not only makes the operation more complicated but also may cause extra decoherence. In addition, the proposal in Ref. [6] employed the resonant coupling of the microwave pulses with the SQUIDs, therefore one needs a precise control of the qubits' level spacings in order to match the microwave frequency.

In this paper, we propose a significantly improved approach to achieve entanglement, information transfer, CNOT gate, and SWAP gate with three-level $\Lambda$-type SQUID qubits in cavity QED. As shown below, the above mentioned problems in Ref. [6] are all mitigated in the present proposal. 
Let us first introduce the Hamiltonian of a SQUID qubit coupled to a single-mode cavity field and a classical microwave pulse with $\mathbf{B}_{\mu w}(\mathbf{r}, t)=\mathbf{B}_{\mu w}(\mathbf{r}) \cos \omega_{\mu w} t$. Here, $\mathbf{B}_{\mu w}(\mathbf{r})$ is the amplitude of the magnetic component and $\omega_{\mu w}$ is the carrier frequency. The qubits considered in this letter are rf SQUIDs each consisting of a Josephson tunnel junction in a superconducting loop (typical size of an rf SQUID is on the order of $10 \mu m-100 \mu m$ ). The Hamiltonian of an rf SQUID (with junction capacitance $C$ and loop inductance $L$ ) can be written in the usual form

$$
H_{s}=\frac{Q^{2}}{2 C}+\frac{\left(\Phi-\Phi_{x}\right)^{2}}{2 L}-E_{J} \cos \left(2 \pi \frac{\Phi}{\Phi_{0}}\right)
$$

where $\Phi$, the magnetic flux threading the ring, and $Q$, the total charge on the capacitor, are the conjugate variables of the system (with the commutation relation $[\Phi, Q]=i \hbar$ ), $\Phi_{x}$ is the static (or quasistatic) external flux applied to the ring, and $E_{J} \equiv I_{c} \Phi_{0} / 2 \pi$ is the maximum Josephson coupling energy $\left(I_{c}\right.$ is the critical current of the junction and $\Phi_{0}=h / 2 e$ is the flux quantum).

The quantized Hamiltonian of the cavity mode is given by $H_{c}=\hbar \omega_{c}\left(c^{+} c+1 / 2\right)$, where $c^{+}$and $c$ are the photon creation and annihilation operators; and $\omega_{c}$ is the frequency of the cavity mode.

Consider a $\Lambda$-type configuration formed by the two lowest levels and an excited level of the SQUID, denoted by $|0\rangle,|1\rangle$ and $|a\rangle$ with energy eigenvalues $E_{0}, E_{1}$, and $E_{a}$, respectively [Fig. 1(a)]. For the sake of concreteness, we choose the following device and control parameters: $C=90 \mathrm{fF}, L=100 \mathrm{pH}, I_{c}=3.75 \mu \mathrm{A}, \Phi_{x}=0.4995 \Phi_{0}$ for the SQUID qubit in the rest of this letter. We can show that when the cavity mode is coupled to the $|0\rangle \leftrightarrow|a\rangle$ transition but far-off resonant with the $|0\rangle \leftrightarrow|1\rangle$ and $|1\rangle \leftrightarrow|a\rangle$ transitions, and when the microwave pulse is coupled to the $|1\rangle \leftrightarrow|a\rangle$ transition while far-off resonant with the $|0\rangle \leftrightarrow|1\rangle$ and $|0\rangle \leftrightarrow|a\rangle$ transitions, the Hamiltonian of the system can be written as:

$$
\begin{aligned}
H=E_{0} \sigma_{00} & +E_{1} \sigma_{11}+E_{a} \sigma_{a a}+\hbar \omega_{c} c^{+} c \\
& +\hbar\left(g c^{+} \sigma_{0 a}+\text { h.c. }\right)+\hbar\left(\Omega e^{i \omega_{\mu w} t} \sigma_{1 a}+\text { h.c. }\right)
\end{aligned}
$$


where $g$ is the coupling constant between the cavity mode and the $|0\rangle \leftrightarrow|a\rangle$ transition; $\Omega$ is the Rabi-flopping frequency corresponding to the $|1\rangle \leftrightarrow|a\rangle$ transition; and $\sigma_{i j}=|i\rangle\langle j|$ $(i, j=0,1, a)$. The expressions of $g$ and $\Omega$ are given by

$$
\begin{aligned}
& g=\frac{1}{L} \sqrt{\frac{\omega_{c}}{2 \mu_{0} \hbar}}\langle 0|\Phi| a\rangle \int_{S} \mathbf{B}_{c}(\mathbf{r}) \cdot d \mathbf{S}, \\
& \Omega=\frac{1}{2 L \hbar}\langle 1|\Phi| a\rangle \int_{S} \mathbf{B}_{\mu w}(\mathbf{r}) \cdot d \mathbf{S},
\end{aligned}
$$

where $S$ is any surface that is bounded by the SQUID ring, $\mathbf{r}$ is the position vector on $S$, and $\mathbf{B}_{c}(\mathbf{r})$ is the magnetic component of the normal mode of the cavity. For a standing-wave cavity, one has $\mathbf{B}_{c}(z)=\mu_{0} \sqrt{\frac{2}{V}} \cos \mathrm{k} z$ (here, $\mathrm{k}$ is the wave number, $V$ and $z$ are the cavity volume and the cavity axis, respectively).

Consider a situation in which the cavity mode is largely detuned from the $|0\rangle \leftrightarrow|a\rangle$ transition, i.e., $\Delta_{c}=\omega_{a 0}-\omega_{c} \gg g$, and the microwave pulse is largely detuned from the $|1\rangle \leftrightarrow|a\rangle$ transition, i.e., $\Delta_{\mu w}=\omega_{a 1}-\omega_{\mu w} \gg \Omega$, where $\omega_{a 0}=\left(E_{a}-E_{0}\right) / \hbar$ and $\omega_{a 1}=\left(E_{a}-E_{1}\right) / \hbar[$ Fig. 1(a)]. Under this condition, the intermediate level $|a\rangle$ can be adiabatically eliminated $[10,11]$. Thus, the effective Hamiltonian in the interaction picture becomes $[10,11]$

$$
H_{i}=\hbar\left[-\frac{g^{2}}{\Delta_{c}} c^{+} c \sigma_{00}-\frac{\Omega^{2}}{\Delta_{\mu w}} \sigma_{11}-g_{e f f} e^{i \delta t} c \sigma_{01}^{+}-g_{e f f} e^{-i \delta t} c^{+} \sigma_{01}\right],
$$

where $\sigma_{01}=|0\rangle\left\langle 1\left|, \sigma_{01}^{+}=\right| 1\right\rangle\langle 0|, \delta=\Delta_{c}-\Delta_{\mu w}$, and $g_{e f f}=\frac{\Omega g}{2}\left(\frac{1}{\Delta_{c}}+\frac{1}{\Delta_{\mu w}}\right)$. The first two terms are ac-Stark shifts of the levels $|0\rangle$ and $|1\rangle$ induced by the cavity mode and the microwave pulse, respectively. The last two terms are the familiar Jaynes-Cummings interaction, describing the Raman coupling of the two lowest levels of the SQUID.

Effective Hamiltonian for two SQUID qubits in cavity. To simplify presentation, let us consider two identical SQUIDs $I$ and $I I$ ( the method is also applicable to non-identical SQUIDs). The two SQUIDs are coupled to the same single-mode microwave cavity and each driven by a classical microwave pulse $\mathbf{B}_{\mu w}^{i}(\mathbf{r}, t)=\mathbf{B}_{\mu w}^{i}(\mathbf{r}) \cos \omega_{\mu w} t(i=I, I I)$ [Fig. 2]. The separation of the two SQUIDs is assumed to be much larger than the linear dimension of each SQUID ring in such a way that direct interaction between the two SQUIDs is negligible. 
Also, suppose that the coupling of each SQUID to the cavity mode is the same (this can be readily obtained by placing the two squbits at two locations $\mathbf{r}_{1}$ and $\mathbf{r}_{2}$ of the cavity axis where the magnetic fluxes generated by the cavity field are the same). In this case, it is obvious that based on Eq. (4), the Hamiltonian for the system in the interaction picture can be written as

$$
\begin{aligned}
H_{I}= & \sum_{i=I, I I} \hbar\left[-\frac{g^{2}}{\Delta_{c}} c^{+} c \sigma_{00 i}-\frac{\Omega^{2}}{\Delta_{\mu w}} \sigma_{11 i}\right] \\
& -\hbar \sum_{i=I, I I}\left[g_{e f f} e^{i \delta t} c \sigma_{01 i}^{+}+g_{e f f} e^{-i \delta t} c^{+} \sigma_{01 i}\right] .
\end{aligned}
$$

Under the condition that $\delta \gg \frac{g^{2}}{\Delta_{c}}, \frac{\Omega^{2}}{\Delta_{\mu w}}, g_{e f f}$, there is no exchange of energy between the SQUIDs and the cavity mode. The effective Hamiltonian is then given by [12-15]

$$
\begin{aligned}
H_{e f f}=\sum_{i=I, I I} \hbar\left[-\frac{g^{2}}{\Delta_{c}} c^{+} c \sigma_{00 i}-\frac{\Omega^{2}}{\Delta_{\mu w}} \sigma_{11 i}\right] & \\
& +\hbar \gamma\left[\sum_{i=I, I I}-c^{+} c \sigma_{00 i}+c c^{+} \sigma_{11 i}+\sigma_{01 I}^{+} \sigma_{01 I I}+\sigma_{01 I} \sigma_{01 I I}^{+}\right]
\end{aligned}
$$

where the third and fourth terms describe the photon-number dependent Stark shifts induced by the off-resonant Raman coupling, and the last two terms describe the "dipole" coupling between the two SQUIDs mediated by the cavity mode and the classical fields. The parameter $\gamma=g_{\text {eff }}^{2} / \delta$ characterizes the strength of Stark shift and inter-qubit coupling. If the cavity is initially in the vacuum state, then the effective Hamiltonian reduces to

$$
H_{e f f}=-\sum_{i=I, I I} \hbar \frac{\Omega^{2}}{\Delta_{\mu w}} \sigma_{11 i}+\hbar \gamma\left[\sum_{i=I, I I} \sigma_{11 i}+\sigma_{01 I}^{+} \sigma_{01 I I}+\sigma_{01 I} \sigma_{01 I I}^{+}\right],
$$

Note that the Hamiltonian (7) does not contain the operators of the cavity mode. Thus, only the state of the SQUID system undergoes an evolution under the Hamiltonian (7), i.e., no quantum information transfer occurs between the SQUIDs and the cavity mode. Therefore, the cavity mode is virtually excited.

The Hamiltonian (7) acting on the system can be expressed through the unitary transformation 


$$
\mathcal{U}(t>0)=\left(\begin{array}{cccc}
1 & 0 & 0 & 0 \\
0 & e^{-i \gamma^{\prime} t} \cos (\gamma t) & -i e^{-i \gamma^{\prime} t} \sin (\gamma t) & 0 \\
0 & -i e^{-i \gamma^{\prime} t} \sin (\gamma t) & e^{-i \gamma^{\prime} t} \cos (\gamma t) & 0 \\
0 & 0 & 0 & e^{-i 2 \gamma^{\prime} t}
\end{array}\right)
$$

in the two-qubit computational subspace $\{|00\rangle,|01\rangle,|10\rangle,|11\rangle\}$, where $\gamma^{\prime}=\gamma-\frac{\Omega^{2}}{\Delta_{\mu w}}$. In the following, based on Eq. (8) we show how quantum entanglement, information transfer, and swap gates can be achieved.

Generation of entanglement. The two logical states of each SQUID qubit are represented by the two lowest energy states $|0\rangle$ and $|1\rangle$. From (8), one can see that if the two SQUID qubits are initially in the states $|0\rangle_{I}$ and $|1\rangle_{I I}$, they will evolve to the following maximally entangled state after an interaction time $\pi /(4 \gamma)$

$$
|\psi\rangle=\frac{1}{\sqrt{2}}\left(|0\rangle_{I}|1\rangle_{I I}-i|1\rangle_{I}|0\rangle_{I I}\right),
$$

where the common phase factor $e^{-i \chi \pi / 4}\left(\chi=\gamma^{\prime} / \gamma\right)$ has been omitted.

Quantum information transfer. Suppose that the SQUID qubit $I$ is the original carrier of quantum information, which is in an arbitrary state $\alpha|0\rangle+\beta|1\rangle$. The quantum information transfer from the qubit $I$ to the qubit $I I$ initially in the state $|0\rangle$ is described by

$$
\left(\alpha|0\rangle_{I}+\beta|1\rangle_{I}\right)|0\rangle_{I I} \rightarrow|0\rangle_{I}\left(\alpha|0\rangle_{I I}+\beta|1\rangle_{I I}\right)
$$

which can be easily realized via only two steps: First, apply two microwave pulses to the two SQUIDs $I$ and $I I$, respectively, so that the states of the two SQUIDs undergo an evolution under the Hamiltonian (7) for an interaction time $\pi /(2 \gamma)$. Then, perform a single-qubit phase shift $|0\rangle \rightarrow e^{-i(1+\chi) \pi / 4}|0\rangle$ while $|1\rangle \rightarrow e^{i(1+\chi) \pi / 4}|1\rangle$ on the SQUID qubit $I I$. The states after each step of the operations are listed below:

$$
\begin{aligned}
& \left(\alpha|0\rangle_{I}+\beta|1\rangle_{I}\right)|0\rangle_{I I} \stackrel{\text { Step (i) }}{\longrightarrow}|0\rangle_{I}\left[\alpha|0\rangle_{I I}+e^{-i(1+\chi) \pi / 2} \beta|1\rangle_{I I}\right] \\
& \stackrel{\text { Step (ii) }}{\longrightarrow} e^{-i(1+\chi) \pi / 4}|0\rangle_{I}\left(\alpha|0\rangle_{I I}+\beta|1\rangle_{I I}\right) .
\end{aligned}
$$


It is clear that the two-step operation transfers quantum information from the SQUID qubit $I$ to the SQUID qubit $I I$.

Single SQUID qubit operations can be achieved without real excitation of the intermediate level $|a\rangle$, by applying two microwave pulses $a$ and $b$ with $\mathbf{B}_{\mu w}^{k}(\mathbf{r}, t)=$ $\mathbf{B}_{\mu w}^{k}(\mathbf{r}) \cos \left(\omega_{\mu w}^{k} t+\phi_{k}\right)(k=a, b)$ in order to induce two-photon Raman resonant transition between the qubit levels $|0\rangle$ and $|1\rangle$ [Fig. 1 (b)]. During the single-qubit operation, the cavity mode can be decoupled from the qubits without adjusting the squbits' level spacings. The reason for this is that one can choose the frequencies of the applied microwave pulses so that two-photon Raman resonant transition between the qubit levels $|0\rangle$ and $|1\rangle$ is satisfied, while the cavity mode is highly detuned from either pulse [see Fig. 1 (b)]. In the case when $\Omega_{a}=\Omega_{b}$, or $\delta \Omega \ll \Omega_{a} \Omega_{b} / \bar{\Omega}$ (here, $\delta \Omega=\Omega_{a}-\Omega_{b}$ and $\bar{\Omega}=\frac{\Omega_{a}+\Omega_{b}}{2}$ ), the single-qubit rotation can be realized as follows

$$
\begin{aligned}
& |0\rangle \rightarrow \cos g^{\prime} t|0\rangle+e^{-i\left(\phi_{a}-\phi_{b}-\pi / 2\right)} \sin g^{\prime} t|1\rangle, \\
& |1\rangle \rightarrow e^{i\left(\phi_{a}-\phi_{b}+\pi / 2\right)} \sin g^{\prime} t|0\rangle+\cos g^{\prime} t|1\rangle,
\end{aligned}
$$

where $g^{\prime}=\Omega_{a} \Omega_{b} / \Delta$; and $\Omega_{a}\left(\Omega_{b}\right)$ is the Rabi flopping frequency between the levels $|0\rangle$ and $|a\rangle$ (the levels $|1\rangle$ and $|a\rangle$ ) generated by the pulse $a(b)$.

From (12), one can see that the above single-qubit phase shift $|0\rangle \rightarrow e^{-i(1+\chi) \pi / 4}|0\rangle$ and $|1\rangle \rightarrow e^{i(1+\chi) \pi / 4}|1\rangle$ can be realized in the following two steps:

Step (i). Apply two microwave pulses $a$ and $b$ to the SQUID with a phase difference $\delta \phi=\phi_{a}-\phi_{b}=(1+\chi) \pi / 4$, so that the states of the SQUID qubit undergo an evolution under (12) for an interaction time $t_{1}=\pi /\left(2 g^{\prime}\right)$.

Step (ii). Apply two microwave pulses $a$ and $b$ to the SQUID with $\phi_{a}-\phi_{b}=\pi$, so that the states of the SQUID qubit undergo an evolution under (12) for another interaction time $t_{2}=t_{1}=\pi /\left(2 g^{\prime}\right)$.

The states after each step of the above operation are summarized below

$$
\begin{aligned}
& |0\rangle \underset{\text { Step (i) }}{\rightarrow} e^{-i(\delta \phi-\pi / 2)}|1\rangle \underset{\text { Step (ii) }}{\longrightarrow} e^{-i \delta \phi}|0\rangle \text {, } \\
& |1\rangle \quad e^{i(\delta \phi+\pi / 2)}|0\rangle \quad e^{i \delta \phi}|1\rangle
\end{aligned}
$$


which implies that the operations described above achieve a single-qubit phase shift $|0\rangle$ $\rightarrow e^{-i \delta \phi}|0\rangle$ and $|1\rangle \rightarrow e^{i \delta \phi}|1\rangle$ with a phase $\delta \phi=(1+\chi) \pi / 4$.

Quantum CNOT gate. A non-trivial and universal two-qubit controlled NOT gate (CNOT) can be realized by combining two-qubit operations with single-qubit operations. We find that the CNOT gate $|i\rangle_{I}|j\rangle_{I I} \rightarrow|i\rangle_{I}|i \oplus j\rangle_{I I}(i, j \in\{0,1\})$ acting on the two SQUID qubits $I$ and $I I$ can be achieved through the following unitary transformations

$$
\mathcal{U}_{C N O T}=\mathcal{H}_{I I}^{-1} U_{I} U_{I I} \mathcal{S}_{I} \mathcal{S}_{I I} \mathcal{U}_{I, I I} U_{I}^{\prime} \mathcal{S}_{I I} \mathcal{U}_{I, I I} \mathrm{H}_{I I} \mathrm{H}_{I} \mathcal{H}_{I I}
$$

where the common phase factor $e^{-i \chi \pi / 4}$ is omitted, the subscripts $I$ and $I I$ represent qubits $I$ and $I I, \mathcal{U}_{I, I I}$ is a two-SQUID-qubit joint unitary operation defined by (8) with $t=\pi /(4 \gamma)$, $\mathcal{S}_{I}$ and $\mathcal{S}_{I I}$ are both single-qubit phase-shift operations each resulting in $|0\rangle \rightarrow e^{-i \chi \pi / 8}|0\rangle$ and $|1\rangle \rightarrow e^{i \chi \pi / 8}|1\rangle, U_{I}=\mathrm{H}_{I}^{-1} \mathcal{H}_{I}, U_{I I}=\mathrm{H}_{I I}^{-1} \mathcal{H}_{I I}^{-1}$, and $U_{I}^{\prime}=\sigma_{y_{I}} \mathcal{S}_{I}$ ( $\sigma_{y}$ is the Pauli operator). In above, $\mathcal{H}, \mathcal{H}^{-1}, \mathrm{H}$, and $\mathrm{H}^{-1}$ are the following Hadamard transformations

$$
\mathcal{H}=\frac{1}{\sqrt{2}}\left(\begin{array}{cc}
1 & -1 \\
1 & 1
\end{array}\right), \mathrm{H}=\frac{1}{\sqrt{2}}\left(\begin{array}{cc}
1 & -i \\
-i & 1
\end{array}\right), \mathcal{H H}^{-1}=\mathrm{HH}^{-1}=\mathrm{I}
$$

in the single-qubit Hilbert subspace formed by $|0\rangle=(0,1)^{\mathrm{T}}$ and $|1\rangle=(1,0)^{\mathrm{T}}$. It is straightforward to show that with a proper choice of $\phi_{a}, \phi_{b}$, and $t$, the four basic single-qubit Hadamard transformations can be obtained from the above single-qubit rotation (12).

Quantum SWAP gate. It is known that constructing a SWAP gate requires at least three CNOT gates as follows [16]

$$
\begin{aligned}
|i\rangle_{I}|j\rangle_{I I} & \rightarrow|i\rangle_{I}|i \oplus j\rangle_{I I} \\
& \rightarrow|i \oplus(i \oplus j)\rangle_{I}|i \oplus j\rangle_{I I}=|j\rangle_{I}|i \oplus j\rangle_{I I} \\
& \rightarrow|j\rangle_{I}|(i \oplus j) \oplus j\rangle_{I I}=|j\rangle_{I}|i\rangle_{I I}
\end{aligned}
$$

where $i, j \in\{0,1\}$ and all additions are done modulo 2. As described above, a CNOT requires 10 single-qubit operations and 2 two-qubit operations. Thus, at least 36 basic operations for three CNOT gates are needed to implement a two-SQUID-qubit SWAP gate 
by using the above method. In the following discussion we present a simple way to perform a SWAP.

Note that the information transfer $(10)$ is equivalent to a transformation $|i\rangle_{I}|0\rangle_{I I} \rightarrow$ $|0\rangle_{I}|i\rangle_{I I}(i \in\{0,1\})$. Thus, a two-SQUID-qubit SWAP $|i\rangle_{I}|j\rangle_{I I} \rightarrow|j\rangle_{I}|i\rangle_{I I}(i, j \in\{0,1\})$ can be realized through the following procedure:

$$
|i\rangle_{I}|j\rangle_{I I}|0\rangle_{a} \rightarrow|0\rangle_{I}|j\rangle_{I I}|i\rangle_{a} \rightarrow|j\rangle_{I}|0\rangle_{I I}|i\rangle_{a} \rightarrow|j\rangle_{I}|i\rangle_{I I}|0\rangle_{a}
$$

i.e., transfer information first from qubit $I$ to the auxiliary qubit $a$; then from qubit $I I$ to qubit $I$; and finally, from the auxiliary qubit $a$ to qubit $I I$. The irrelevant qubit in each step can be decoupled from the cavity field and the other two qubits by turning off the microwave pulse directed to it. As described above, the information transfer from one qubit to another only needs one two-qubit operation plus two basic single-qubit operations for realizing a single-qubit phase-shift. Thus, nine basic operations are sufficient to construct a two-SQUID-qubit SWAP gate.

It is necessary to give a brief description on experimental parameters. For the SQUIDs with the parameters given above and with junction's damping resistance $R>1$ G $\Omega$ [17], the level $|a\rangle$ 's energy relaxation time $T_{1} \simeq \frac{R}{60 \mathrm{M} \Omega} \cdot \mu$ s would be $\sim 15 \mu$ s. The transition frequency is $\omega_{a 0} /(2 \pi) \simeq 30 \mathrm{GHz}$. Hence, we choose $\omega_{c} /(2 \pi)=29.7 \mathrm{GHz}$ as the cavity-mode frequency. For a superconducting standing-wave cavity with a volume $10 \times 1 \times 1 \mathrm{~mm}^{3}$ and a SQUID with a $50 \times 50 \mu \mathrm{m}^{2}$ loop located at one of the antinodes of the $\mathbf{B}$ field, the coupling constant can be calculated using Eq. (3), which gives $g \simeq 1.8 \times 10^{8} \mathrm{~s}^{-1}$, i.e., about $0.1 \Delta_{c}$. By choosing the frequency and amplitude of the microwave pulse appropriately such that $\Delta_{\mu w}=10 \Omega$ and $g=1.2 \Omega$ for each SQUID, we have $\delta \simeq 10 g_{\text {eff }} \simeq 3.1 \times 10^{8} \mathrm{~s}^{-1}$, i.e., $\gamma=g_{\text {eff }}^{2} / \delta \simeq 3.1 \times 10^{6}$ $\mathrm{s}^{-1}$. Then the time needed for creating the entangled state (9) or the typical SQUID-cavity interaction time (the time for each two-SQUID-qubit joint operation) required for CNOT gate is $T_{s-c}=\pi /(4 \gamma) \simeq 0.25 \mu \mathrm{s}$, and the typical SQUID-cavity interaction time needed for quantum information transfer or SWAP gate is $T_{s-c}^{\prime}=\pi /(2 \gamma) \simeq 0.5 \mu \mathrm{s}$, which are much shorter than the level $|a\rangle$ 's effective decay time $T_{1} / P_{a} \geq 1.5 \times 10^{3} \mu$ s for $T_{1}=15$ 
$\mu \mathrm{s}$, where $P_{a} \leq 0.01$ is the occupational probability of the level $|a\rangle$ for the present case. The photon lifetime is given by $T_{c}=Q_{c} / \omega_{c}$ where $Q_{c}$ is the quality factor of the cavity. In the present case, the cavity has a probability $P_{c} \simeq 0.01$ of being excited during the operation. Thus, the effective decay time of the cavity is $T_{c} / P_{c} \simeq 10 \mu \mathrm{s} \gg T_{s-c}, T_{s-c}^{\prime}$ for $Q_{c}=2 \times 10^{4}$, which is realizable for superconducting microwave cavities since recent experiments have demonstrated that the quality factor of microwave cavities loaded with thin film superconducting circuits (on single crystal $\mathrm{Si}$ or $\mathrm{MgO}$ substrates) are greater than $10^{4}[7-9,18]$ and a superconducting microwave cavity with a $Q>10^{6}$ has been also reported more recently [19].

In addition to the advantages of the scheme described in Ref. [6], i.e., virtually excitation of the cavity mode and no tunneling between the qubit levels $|0\rangle$ and $|1\rangle$ being needed, the present scheme has the following distinct features: (i) During the operation, the intermediate level is virtually excited and thus the operation errors caused by energy relaxation is greatly suppressed. (ii) No adjustment of level spacings is needed during operations, since the qubitqubit interaction required for the two-qubit operations is via the cooperative actions of the cavity mode and the microwave pulses. (iii) The method does not require two SQUIDs with identical parameters, since in the case of non-identical SQUIDs $I$ and $I I$, one has $\delta_{I}=\omega_{a 0}^{I}-\omega_{a 1}^{I}-\omega_{c}+\omega_{\mu w}^{I}$ and $\delta_{I I}=\omega_{a 0}^{I I}-\omega_{a 1}^{I I}-\omega_{c}+\omega_{\mu w}^{I I}$, which can always be set to equal by adjusting the frequencies, $\omega_{\mu w}^{I}$ and $\omega_{\mu w}^{I I}$, of the two microwave pulses applied to the SQUIDs. Thus, the present scheme can allow finite device parameter non-uniformity. (iv) The method could be extended to perform QIP on many SQUID qubits in a cavity. In the present case, SQUID qubits are chosen by the application of the microwave pulses, i.e., those SQUIDs who are unaddressed by the microwave pulses are not involved in the operation. However, in the scheme of [6], one has to adjust SQUID level spacings to have SQUID qubits to be coupled or decoupled.

Before we conclude, we should mention that coupling qubits via cavity/trap-assisted collision without real excitation of the cavity/vibrational mode was previously reported in Ref. [15] and that the type of effective Hamiltonian (6) or (7), obtained after the adiabatic 
elimination of both the excited states of the SQUIDs and the cavity mode, was proposed previously for a trapped-ion based quantum processor [15] or atom-cavity based quantum processor $[12,13]$. However, we point out that the main purpose of this work is to show that the same interaction model can be obtained in a solid-state system in which the qubits are embodied by rf SQUIDs. We think that this result is interesting by itself as a consequence of the analogy between a superconducting qubit and an atom/ion under proper conditions.

In summary, we have proposed a theoretical method for realizing quantum entanglement, information transfer, CNOT gate, and SWAP gate with SQUID qubits in cavity. It is shown that the approach is robust against important sources of decoherence due to energy relaxation of the intermediate level. We stress that in all above analysis, all Stark shift terms, which might affect the operation fidelity, are included. In addition, we have shown that the realization of the scheme is possible within the present technique. To the best of our knowledge, there has been no experimental demonstration of entanglement, information transfer or logical gates for the SQUID systems. Therefore, we hope that this work will stimulate further theoretical and experimental activities in this emerging research field.

CPY is very grateful to Prof. Shi-Biao Zheng for many fruitful discussions and very useful comments. This research was partially supported by National Science Foundation QuBIC program (ECS-0201995), and AFOSR (F49620-01-1-0439), funded under the Department of Defense University Research Initiative on Nanotechnology (DURINT) Program and by the ARDA. 


\section{REFERENCES}

${ }^{1}$ T. Sleator and H. Weinfurter, Phys. Rev. Lett. 74, 4087 (1995).

2 J. I. Cirac and P. Zoller, Phys. Rev. Lett. 74, 4091 (1995).

${ }^{3}$ M. S. Sherwin et. al., Phys. Rev. A 60, 3508 (1999).

${ }^{4}$ A. Blais, R. S. Huang, A. Wallraff, S. M. Girvin, and R. J. Schoelkopf, LANL eprint, cond-mat/0402216.

${ }^{5}$ X. X. Yi, X. H. Su, and L. You, Phys. Rev. Lett. 90, 097902 (2003).

${ }^{6}$ C. P. Yang, S. I. Chu, and S. Han, Phys. Rev. A 67, 042311 (2003).

${ }^{7}$ D. Vion et al., Science 296, 886 (2002).

${ }^{8}$ Y. Yu, S. Han, X. Chu, S. I. Chu, and Z. Wang, Science 296, 889 (2002).

${ }^{9}$ I. Chiorescu et. al., Science 299, 1869 (2003).

${ }^{10}$ L. Wang, R. R. Puri, and J. H. Eberly, Phys. Rev. A 46, 7192 (1992).

${ }^{11}$ L. Wang and J. H. Eberly, Phys. Rev. A 47, 4248 (1993).

12 S. B. Zheng and G. C. Guo, Phys. Rev. Lett. 85, 2392 (2000); S. B. Zheng, Phys. Rev. Lett. 87, 230404 (2001).

${ }^{13}$ S. B. Zheng, L. H. Lin, and Y. K. Jiang, Communications in Theoretical Physics 40, 103 (2003).

${ }^{14}$ G. S. Agarwal, R. R. Puri, and R. P. Singh, Phys. Rev. A 56, 2249 (1997).

15 A. Sørensen and K. Mølmer, Phys. Rev. Lett. 82, 1971 (1999).

${ }^{16}$ M. A. Nielsen and I. L. Chuang, Quantum Computation and Quantum Information, (Cambridge University Press, Cambridge, England, 2001).

17 J. E. Lukens, Private communication, (2003). 
${ }^{18}$ R. J. Schoelkopf, DURINT annual review meeting, New York City, May 2003.

${ }^{19}$ P. K. Day, H. G. LeDuc, B. Mazin, A. Vayonakis, J. Zmuidzinas, Nature (London) 425, 817 (2003). 


\section{Figure Captions}

FIG. 1. (a) The potential and level diagram of an rf SQUID with a $\Lambda$-type three levels $|0\rangle,|1\rangle$ and $|a\rangle$. The cavity field is detuned from the classical microwave pulse by $\delta=$ $\Delta_{c}-\Delta_{\mu w}$. (b) Illustration of single-qubit operation. The two microwave pulses $a$ and $b$ with frequencies $\omega_{\mu w}^{a}$ and $\omega_{\mu w}^{b}$ are applied to induce two-photon Raman resonant transition between the qubit levels $|0\rangle$ and $|1\rangle$ with $\omega_{10}=\omega_{\mu w}^{a}-\omega_{\mu w}^{b}$, for the purpose of single-qubit logic operation. To have the intermediate level $|a\rangle$ not populated during the operation, the setting $\Delta=\omega_{a 0}-\omega_{\mu w}^{a}=\omega_{b 0}-\omega_{\mu w}^{b} \gg \Omega_{a}, \Omega_{b}$ should be met, where $\Omega_{a}\left(\Omega_{b}\right)$ is the Rabi flopping frequency between the levels $|0\rangle$ and $|a\rangle$ (the levels $|1\rangle$ and $|a\rangle$ ) generated by the pulse $a(b)$.

FIG. 2. Schematic illustration of two SQUIDs $(I, I I)$ coupled to a single-mode cavity field and manipulated by microwave pulses. The two SQUIDs are placed along the cavity axis (the $Z$ axis) and in the $X-Z$ plane.The two SQUIDs are placed along the cavity axis (the $Z$ axis) and in the $X-Z$ plane. $\mathbf{B}_{c}, \mathbf{B}_{\mu w}^{I}$ and $\mathbf{B}_{\mu w}^{I I}$ are in $Y$ direction. 


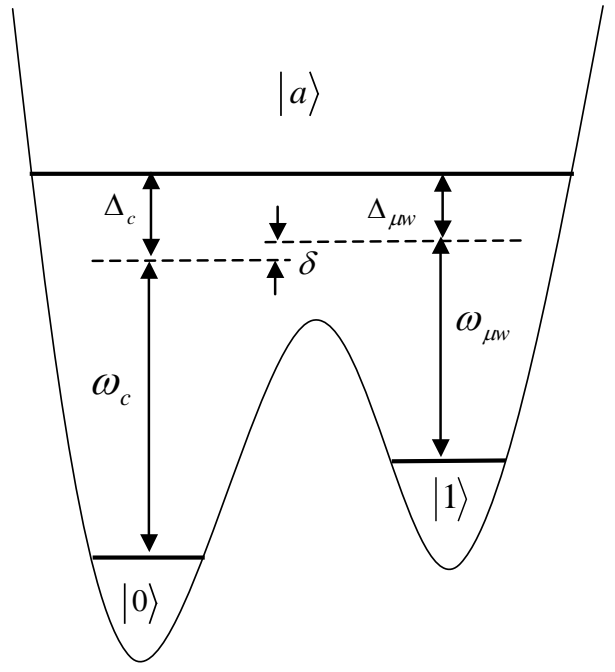

(a)

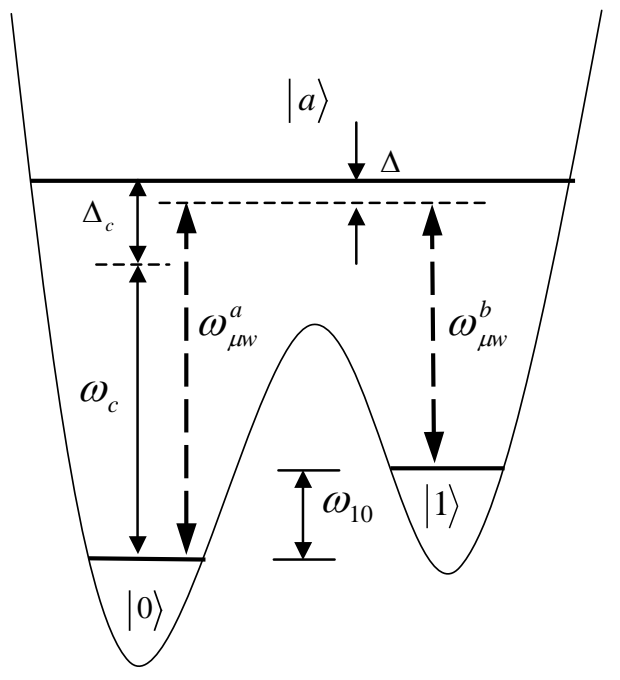

(b)

FIG. 1 

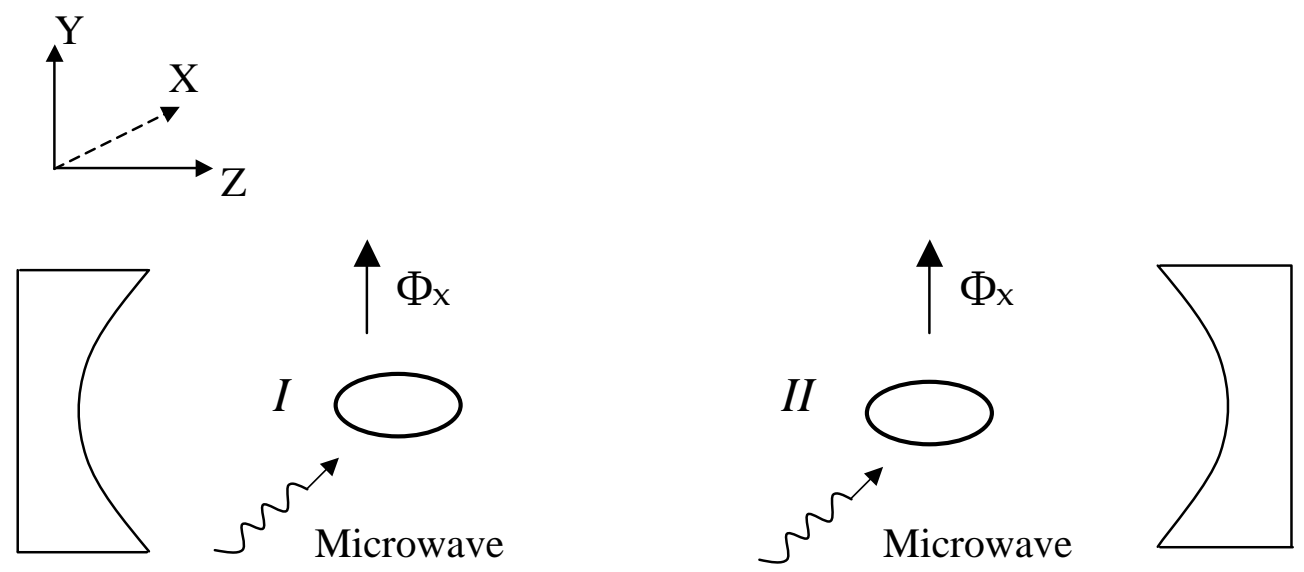

FIG. 2 